\title{
LIX. The eye as an electrical organ
}

\section{W.M. Thornton D.Sc. D.Eng.}

To cite this article: W.M. Thornton D.Sc. D.Eng. (1910) LIX. The eye as an electrical organ , Philosophical Magazine Series 6, 20:118, 560-563, DOI: 10.1080/14786441008636938

To link to this article: http://dx.doi.org/10.1080/14786441008636938

册 Published online: 21 Apr 2009.

Submit your article to this journal 준

Џll Article views: 3

Q View related articles $₫$ 


\section{$\left[\begin{array}{ll}560 & ]\end{array}\right.$}

LIX. The Eye as an Electrical Organ. By W. M. Thonston, D.Sc., D.Eng., Professor of Electrical Engineering, Armstrong College, Neweastle-on-Tyne* .

1. $\mathrm{N}$ the electromagnetic theory of light the amplitude of a progressive wave in a partially conducting medium is reduced by dissipation of energy as heat. The optical media of the eye are typical conducting dielectrics, and the following notes are a consideration of (1) the influence of absorption on vision, (2) the reticulation of the optic nerve on the inner surface of the retina, (3) the least electrical current which can be detected by the eye as light, from the point of view of the electromagnetic theory. Apart from the physiological question of the degree in which sensation is proportional to stimulus, and considering only the energy of the wave, the reduction of light in intensity between entering the cornea and reaching the retina can be found by the expressions given by Maxwell $\dagger$ and Heaviside $\ddagger$ for the decrease of amplitude of waves in a conducting dielectric.

In a distance $r$ they are reduced in the ratio $e^{-\alpha r}$, where

$$
\alpha=\frac{1 \cdot 41 \pi}{\lambda}\left[\left\{1+\left(\frac{2 v^{2}}{\rho n}\right)^{2}\right\}^{\frac{1}{2}}-1\right]^{\frac{1}{2}}
$$

in which $\lambda$ is the wave-length, $n$ the frequency, and $v$ the velocity of the wave in the medium of specific resistance $\rho$.

At high frequencies, such as those of light, this reduces to

$$
\alpha=2 \pi v / \rho \text {. }
$$

In the vitreous humour for example, with $\rho=83 \times 10^{9}$ c.G.s. units, and with the velocity of the wave, calculated from the refractive index, equal to $2 \cdot 3 \times 10^{10} \mathrm{~cm}$. a second, $\alpha$ is found to be $1 \cdot 7$, and the reduction of amplitude in $1.43 \mathrm{~cm}$., the thickness of the vitreous humour in the human eye, is to 088 of that of the incident wave. The energy of the wave, which is proportional to the square of the amplitude, is therefore 0077 of the initial value.

The following table gives the thickness and resistivity of the various media, the former for the human eye, the latter for ox eyes, and the reduction of amplitude in each part.

* Communicated by the Author.

+ Electricity and Magnetism, vol. ii. $\$ 798$.

t Electrical Papers, vol. ii. p. 422. See also Lodge, Phil. Mag. April 1899, "On Opacity." 
The resistances were measured by the Kohlrausch bridgetelephone method on freshly killed, though cold, eyes.

The high conductivity of the humours, greater than that of blood, is remarkable.

\begin{tabular}{|c|c|c|c|c|c|c|}
\hline Medium. & Thickness $r$. & $\rho$. & Mean. & $\alpha$. & ar. & $\begin{array}{c}\text { Reduction } \\
\text { ratio. }\end{array}$ \\
\hline Cormea ........... & $\cdot 115 \mathrm{~cm}$. & $\mathbf{4 5 5}$ & $\mathbf{4 5 5}$ & $0 \cdot 3$ & .0345 & $\cdot 968$ \\
Aquenus humour. & $\cdot 36$ & $92-114$ & 103 & $1 \cdot 37$ & $\cdot 495$ & $\cdot 610$ \\
Crystalline lens... & .39 & $650-750$ & 700 & $0 \cdot 20$ & $\cdot 078$ & .925 \\
Vitresus humour. & $1 \cdot 43$ & $80-87$ & 83 & $1 \cdot 7$ & $2 \cdot 43$ & $\cdot 088$ \\
\hline
\end{tabular}

The total reduction of amplitude is the product of all the figures in the last column and is equal to $\cdot 048$; the energy is therefore $\cdot 0023$ of that of the incident light.

The reduction of intensity is independent of frequency, that is, of colour. The limitation of vision at the blue end of the spectrum is not then due to simple absorption of the kind considered, but either to selective absorption-probably in the cornea-or to photo-chemical inactivity of the visual yellow and purple in ultra-violet light.

The greater part of the incident energy is absorbed before reaching the vitreous humour, but the rate of diminution in the latter is so great, that in the case of exposure to very strong light, injury is lessened. The chief function of the vitreous humour, from this point of view, is to act as an absorbing screen protecting the retina from possible overexposure.

Although absorption would appear to reduce the sensitiveness of the eye by its presence, the structure of the retina can by it be much more delicate without risk of injury.

2. Light falling on the retina encounters first the reticulated optic nerve. The reason for the fine subdivision of this is not fully known. The size of the fibres is, however, such that they are peculiarly well suited, as a bolometer, to receive the incident energy.

The distribution of alternating current in the cross-section of a cylindrical conductor is not uniform when the frequency exceeds a certain value, which depends upon the conductivity and diameter. Since the current density is then greater at the circumference, this is known as the "skin effect"; it reduces the effective current in the conductor.

The diameter of the nerve fibres of the retina is such Phil. Mag. S. 6. Vol. 20. No. 118. Oct. 1910. 2 P 
(about 4 to $10 \mu$ ), that notwithstanding the frequency approaching $10^{15}$ a second, the skin effect does not occur. That is, the current density of the electrical current forming the light-wave, which being transverse traverses the fibres longitudinally, is uniform over their cross-section. In the calculation of this the resistivity of the fibres was taken to be, as a lower limit, $20 \mathrm{ohms}$ per cm. cube. The value given by Waller* is $200 \mathrm{ohms}$ per $\mathrm{cm}$. cube for muscle and nerve; the previous value was chosen to cover any possible increase of conductivity in non-medullated fibres. If the inner layer of nerve fibre were continuous and of the same thickness as the fibres, it would not be opaque to electric radiation at the frequency of light, unless by selective absorption. The object of the reticulation cannot therefore be only to let the light through to the rods and cones.

From observations given later, it can be shown that the energy absorbed in the faintest visible light is not sufficient to account for a rise of temperature in the fibres of more than $10^{-8}$ degree $\mathrm{C}$. a second ; and since the eye is able to follow rapid flicker, it seems improbable that the visual stimulus can be in any way thermal in a medium maintained at blood-heat. It is more probable, and it is suggested here, that the stimulus caused by the electrical currents in the light-wave incident on the fibres may be contributory to vision by acting as a continuous "messenger" to the brain, or as the vibrator in a coherer system keeping sensitive the contact at the synapse between the retina and the rods and cones.

3 . An approximate estimate of the least current which can be detected by the eye as light, may be made by considering the distance at which the sun would cease to be visible. The mean energy reaching the earth's surface in full sunlight was found by the late Prof. S. P. Langley to be $4 \cdot 3 \times 10^{-5}$ erg per cubic centimetre. In a letter from him, shortly before his death, he estimates the energy in the visible part of the spectrum from the curves of luminosity to be 21 per cent. of the whole. The stellar magnitude of the sun according to Pickering is -25.5 . The mean of Wollaston's, Zollner's, and Bond's values is -26.4 . Thus with the former value the sun has $4 \times 10^{12}$ the intensity of a star of the 6th magnitude, the highest visible by the unaided eye, with the latter value $9 \cdot 1 \times 10^{12}$. Taking the former as probably the more accurate, the sun would cease to be visible at $2 \times 10^{6}$ its present distance $\dagger$.

The energy reaching the earth would then be $0.25 \times 10^{-12}$

* A. D. Waller, ' Signs of Life.'

† I am indebted to Prof. R. A. Sampson for the astronomical data. 
of Langley's value, that is $1.07 \times 10^{-17} \mathrm{erg}$ per cubic centimetre or 0.32 micro-erg per square centimetre per second. The ratio of the visible to total energy can scarcely be the same at the very low intensities. Retaining it in the absence of more reliable data, the energy in the visible light is -067 micro-erg per square $\mathrm{cm}$. per second. It is then reduced by absorption in the eye in the ratio 0023 , giving $1.5 \times 10^{-4}$ erg, or with a velocity of $2 \cdot 3 \times 10^{10}$ centimetres a second, $6.5 \times 10^{-15} \mathrm{erg}$ per cubic centimetre of space at the retina.

The average energy in unit volume of light-wave in nonmagnetic media is $4 \pi i^{2}$, where $i$ is the root-mean-square value, of the current per square centimetre. The current corresponding to the energy in the visible part of the spectrum is then

$$
i=\left(6.5 \times 10^{-15} / 4 \pi\right)^{\frac{3}{5}}
$$

that is 23 micro-amperes per square centimetre at right angles to the wave front.

Since there is no skin effect, this is also the currentdensity in the nerve fibres. The current in a fibre of $0004 \mathrm{~cm}$. diameter would be $2.87 \times 10^{-14}$ ampere. This, then, appears to be about the least electrical current in a nerve fibre which can produce the sensation of light.

The current, when viewing white clouds in full sunlight, with one's back to the sun, is about $7 \cdot 0 \times 10^{-12}$ ampere in the fibres.

Taking the value of $200 \mathrm{ohms}$ per centimetre cube as the resistivity of nerve, the energy absorbed per centimetre length corresponding to a current of $2 \cdot 87 \times 10^{-14}$ ampere is $1.31 \times 10^{-18}$ watt, or $1.31 \times 10^{-11} \mathrm{erg}$ per centimetre of fibre per second. If each nerve fibre conveys a separate stimulus, this is what may be called the least specific stimulus required in the mental process of vision; the actual length of fibre in the retinal image is a small fraction of a centimetre.

Rayleigh * has suggested that the least power required for hearing is not very different from that of least vision. From Rayleigh's figures for sound, Lodge $\dagger$ finds this power to be 6 micro-ergs per second per square $\mathrm{cm}$. Comparing this with the energy entering the eye at least visibility, obtained above from Langley's values, viz. 0.32 micro-erg per second per square $\mathrm{cm}$., the eye would appear to be able to detect about one twentieth of the energy required for the least perception of sound.

* 'Sound,' Article 384, footnote.

+ Jour. Inst. Elec. Engineers, vol. xxvii. p. 931. 